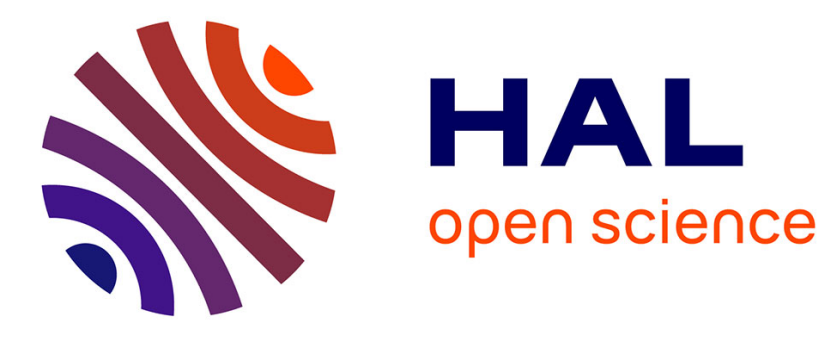

\title{
Validation of a two-fluid model on unsteady water-vapour flows
}

\author{
Fabien Crouzet, Frédéric Daude, Pascal Galon, Jean-Marc Hérard, Olivier \\ Hurisse, Yujie Liu
}

\section{- To cite this version:}

Fabien Crouzet, Frédéric Daude, Pascal Galon, Jean-Marc Hérard, Olivier Hurisse, et al.. Validation of a two-fluid model on unsteady water-vapour flows. Computers and Fluids, 2015, 119, pp.131-142. 10.1016/j.compfluid.2015.06.035 . hal-01242983

\section{HAL Id: hal-01242983 \\ https://hal.science/hal-01242983}

Submitted on 14 Dec 2015

HAL is a multi-disciplinary open access archive for the deposit and dissemination of scientific research documents, whether they are published or not. The documents may come from teaching and research institutions in France or abroad, or from public or private research centers.
L'archive ouverte pluridisciplinaire HAL, est destinée au dépôt et à la diffusion de documents scientifiques de niveau recherche, publiés ou non, émanant des établissements d'enseignement et de recherche français ou étrangers, des laboratoires publics ou privés. 


\title{
Validation of a two-fluid model on unsteady water-vapour flows
}

\author{
Fabien Crouzet, Frédéric Daude, Pascal Galon, \\ Jean-Marc Hérard, Olivier Hurisse, Yujie Liu \\ Fabien Crouzet, Frédéric Daude, Yujie Liu: \\ EDF R\&D, AMA, and LAMSID, UMR EDF/ CNRS/ CEA 8193, \\ 1 avenue du Général de Gaulle, F-92141 Clamart \\ (fabien.crouzet@edf.fr, frederic.daude@edf.fr, yujie.liu@edf.fr) \\ Pascal Galon: \\ CEA Saclay, DEN/DM2S/SEMT/DYN, F-91191 Gif sur Yvette, \\ and LAMSID, UMR EDF/ CNRS/ CEA 8193, \\ 1 avenue du Général de Gaulle, F-92141 Clamart \\ (pascal.galon@cea.fr) \\ Jean-Marc Hérard, Olivier Hurisse: \\ EDF R\&D, MFEE, 6 quai Watier, F-78400 Chatou \\ (jean-marc.herard@edf.fr,olivier.hurisse@edf.fr)
}

\section{Keywords:}

Two-phase flows/ two-fluid model / entropy inequality / water-hammer / vaporization / shock waves / rarefaction waves / validation.

\footnotetext{
Abstract:

This paper is devoted to the validation of a two-fluid two-phase flow model in some highly unsteady situations involving strong rarefaction waves and shocks in watervapour flows. The two-fluid model and its associated numerical method that were introduced in a previous work are first recalled, and details on the computational scheme and the verification of interfacial mass transfer terms are provided. Consistency with experimental data is checked in three configurations. First, a comparison with the speed of sound in a two-phase mixture is detailed. Afterwards, numerical approximations obtained with the two-fluid approach are discussed and compared with some experimental data documented in the Simpson water-hammer experiment and the high depressurization with flashing associated with Canon experiment.
} 


\section{Introduction}

The development of models and associated numerical methods for the simulation of two-phase flows should be achieved in three distinct but evolutionary steps. The derivation of suitable models, both from a mathematical and physical point of view, is the first step that provides closed sets of equations involving non linear PDEs. Then numerical algorithms must be found that would provide convergent series of approximations towards solutions of the latter PDEs, and this corresponds to the verification process. Afterwards numerical results obtained with that set of PDEs must be compared with available experimental data, making sure that the mesh size is sufficiently small so that numerical approximations are no longer sensitive to a further mesh refinement. This last step is referred as the validation step; it is mandatory and is in fact the main objective of the whole approach. Once these three steps have been achieved with the most intense scrutiny, one may tackle the difficult problem of the quantification of uncertainities, but it would be meaningless to begin that work before the modeling / verification / validation steps had been completed, as recalled in [5] for instance.

We focus in this paper on water-vapour flows with mass transfer, with emphasis on water-hammer flows and thus on shock waves occuring in the transient, and on sudden depressurizations that might arise if some loss of fluid would happen in a coolant circuit. This of course requires the application of a two-phase flow model that can handle heat and mass transfer in highly unsteady situations. Actually, the main aim in the current work is to scrutinize a few available validation test cases of unsteady two-phase flows, and in that sense, this work may be seen as a sequel of the paper [28] where emphasis was put on the presentation of a two-fluid model, together with suitable numerical methods and their verification, while restricting to gas-liquid flows without any mass transfer.

Thus we will first recall and summarize herein the main characteristics concerning the two-fluid two-phase flow model that will be used, and its associated numerical method, and then we will investigate features linked with mass transfer. The two-phase flow model describes the dynamics of seven quantities: the statistical fractions, the mean densities, mean velocities and mean temperatures within each phase. The model and its main properties will be briefly recalled in section 2 . For further details on this class of two-fluid models, the reader is referred to $[4,7,8,10,16,18,19,20,22,24$, $11,29,32,33]$ among others. Afterwards, we will rather quickly provide the main numerical tools that are used in the approximation of the two-fluid model in the Finite Volume code. Basically, the algorithm relies on the use of a fractional step method that complies with the entropy inequality, and it treats separately convective terms and relaxation terms. The most important properties and constraints will be briefly described, and a more detailed discussion on some possible way to cope with mass transfer between phases will follow. Obviously, other approaches might be considered as well, such as those introduced in $[1,2,3,22,40,43]$ for instance. The last section will be devoted to the presentation of three distinct cases and associated comments. 


\section{The two-fluid model}

The derivation of the two-fluid model which is described in the sequel relies on classical statistical averaging and closure laws, following a standard thermodynamical approach, the keystone of which is the entropy inequality. Many details and comments can be found in $[20,29]$, and also in $[16,31,32,24,7]$. We would like to emphasize that :

- this particular model does not take the counterpart of single-phase Reynolds stresses into account (these are neglected). Some possible extensions in that direction are currently examined, but this remains beyond the scope of the present work, which basically aims at investigating some validation test cases;

- instantaneous single-phase equations of state rely of stiffened gas EOS. Thus a straightforward consequence is that averaged EOS may be written exactly as functions of the sole main unknowns (mean pressure, mean internal energy and mean density);

- some high-order statistical correlations involving pressure and velocity fluctuations are neglected. As underlined in [29], some non-trivial closure laws might be accounted for, while keeping the same entropy-entropy flux formulation, following the basic approach of Ristorcelli [38], but we also know that this would render the system of PDEs even more intricate $([6,44])$; hence these extensions have not been examined in detail up to now.

Before going further on, we recall that the main specifications for the model derivation are such that:

- a physically relevant entropy inequality should hold for the smooth solutions of the whole model, including viscous terms and sources;

- the homogeneous model obtained by getting rid of viscous and source contributions should be hyperbolic for physically relevant phasic states (thus for positive densities, positive internal energies and positive statistical void fractions);

- unique and meaningful jump conditions should be associated with the latter homogeneous model.

\subsection{Governing equations}

We use classical notations in this paper. Thus $\alpha_{k}(x, t)$ will denote the statistical void fraction of phase $k=l, v$, so that :

$$
\alpha_{l}(x, t)+\alpha_{v}(x, t)=1
$$

Variables $\rho_{k}, U_{k}, P_{k}$ respectively stand for the mean density, the mean velocity, the mean pressure within phase $k$. We also define partial masses:

$$
m_{k}=\alpha_{k} \rho_{k}
$$

The total mean energy $E_{k}$ within phase $k=l, v$ is defined as:

$$
E_{k}=\rho_{k} \varepsilon_{k}\left(P_{k}, \rho_{k}\right)+\rho_{k} U_{k}^{2} / 2
$$


where the function associated with the mean internal energy $\varepsilon_{k}$ only depends on the mean pressure and the mean density $\left(P_{k}, \rho_{k}\right)$.

We can now introduce the set of governing equations for the main unknown $W$ :

$$
W^{t}=\left(\alpha_{v}, m_{l}, m_{v}, m_{l} U_{l}, m_{v} U_{v}, \alpha_{l} E_{l}, \alpha_{v} E_{v}\right)
$$

These governing equations of the two-fluid model read, for $k=l, v$ :

$$
\begin{aligned}
& \partial_{t}\left(\alpha_{v}\right)+V_{i n t}(W) \partial_{x}\left(\alpha_{v}\right)=\phi_{v}(W) \\
& \partial_{t}\left(m_{k}\right)+\partial_{x}\left(m_{k} U_{k}\right)=\Gamma_{k}(W) \\
& \partial_{t}\left(m_{k} U_{k}\right)+\partial_{x}\left(m_{k} U_{k}^{2}\right)+\partial_{x}\left(\alpha_{k} P_{k}\right)-\Pi_{i n t}(W) \partial_{x}\left(\alpha_{k}\right)=D_{k}(W)+\Gamma_{k}(W) \bar{U}_{i n t}(W) \\
& \partial_{t}\left(\alpha_{k} E_{k}\right)+\partial_{x}\left(\alpha_{k} U_{k}\left(E_{k}+P_{k}\right)\right)+\Pi_{i n t}(W) \partial_{t}\left(\alpha_{k}\right)=\psi_{k}(W)+\bar{U}_{\text {int }}(W) D_{k}(W)+\Gamma_{k}(W) \bar{H}_{\text {int }}(W)
\end{aligned}
$$

setting: $\bar{U}_{i n t}=\left(U_{l}+U_{v}\right) / 2$, and: $\bar{H}_{i n t}=U_{l} U_{v} / 2$.

As it has been emphasized in [20,29] among other references, admissible closure laws for $\Pi_{\text {int }}(W)$ may be exhibited in order to comply with a physical entropy inequality. In practice, this means that, assuming a convex form for $V_{\text {int }}(W)$ :

$$
V_{\text {int }}(W)=\xi(W) U_{l}+(1-\xi(W)) U_{v} .
$$

the closure law for $\Pi_{\text {int }}(W)$ should be of the form:

$$
\Pi_{\text {int }}(W)=\chi(W) P_{l}+(1-\chi(W)) P_{v}
$$

with:

$$
\chi(W)=\frac{(1-\xi(W)) / T_{l}}{(1-\xi(W)) / T_{l}+\xi(W) / T_{v}}
$$

The latter function $\chi(W)$ depends on the mean temperatures $T_{k}$ which are defined by:

$$
1 / T_{k}=\partial_{P_{k}}\left(S_{k}\right) / \partial_{P_{k}}\left(\varepsilon_{k}\right)
$$

where the $S_{k}\left(P_{k}, \rho_{k}\right)$ denote the mean phasic entropies that must comply with:

$$
c_{k}^{2} \partial_{P_{k}}\left(S_{k}\right)+\partial_{\rho_{k}}\left(S_{k}\right)=0
$$

denoting:

$$
c_{k}^{2}=\left(\partial_{P_{k}}\left(\varepsilon_{k}\left(P_{k}, \rho_{k}\right)\right)\right)^{-1}\left(\frac{P_{k}}{\left(\rho_{k}\right)^{2}}-\partial_{\rho_{k}}\left(\varepsilon_{k}\left(P_{k}, \rho_{k}\right)\right)\right) .
$$

We also set:

$$
\mu_{k}=\varepsilon_{k}+P_{k} / \rho_{k}-T_{k} S_{k}
$$

At this stage, it only remains to define the source terms $\Gamma_{k}(W), D_{k}(W), \psi_{k}(W)$, which respectively stand for the interfacial mass transfer, the drag effects and the interfacial heat transfer, but also the right-hand side in the governing equation of the statistical void fraction $\phi_{k}(W)$. The latter one arises due to the statistical averaging (see $[16,29])$. Obviously we have:

$$
\sum_{k=l, v} \Gamma_{k}(W)=0 \quad ; \quad \sum_{k=l, v} \psi_{k}(W)=0 \quad ; \quad \sum_{k=l, v} D_{k}(W)=0
$$


and also:

$$
\sum_{k=l, v} \phi_{k}(W)=0 .
$$

Closure laws for the former three $\Gamma_{l}(W), D_{l}(W), \psi_{l}(W)$ correspond to classical terms arising in the two-fluid literature, that is:

$$
\begin{array}{rlrl}
\Gamma_{l}(W) & = & \frac{1}{\tau_{\Gamma}(W)} \frac{m_{l} m_{v}}{m_{l}+m_{v}}\left(K_{l v}^{0}\right)^{-1}\left(\mu_{v}(W) / T_{v}-\mu_{l}(W) / T_{l}\right) ; \\
D_{l}(W) & = & \frac{1}{\tau_{U}(W)} \frac{m_{l} m_{v}}{m_{l}+m_{v}}\left(U_{v}-U_{l}\right) ; \\
\psi_{l}(W) & =\frac{1}{\tau_{T}(W)} \frac{m_{l} m_{v} C_{l v}^{0}}{m_{l}+m_{v}}\left(T_{v}-T_{l}\right) .
\end{array}
$$

where $\tau_{U, \Gamma, T}(W)$ respectively denote the velocity / chemical potential / temperature relaxation time scales, and where the positive constants $K_{l v}^{0}, C_{l v}^{0}$ have the same dimension as the heat capacity $C_{v, l}$. For large times -with respect to the relaxation time scales-, these relaxation terms tend to lead physical states towards a triple velocity-chemical potential-temperature equilibrium. The remaining source term $\phi_{l}(W)$ is defined as:

$$
\phi_{l}(W)=\frac{1}{\tau_{P}(W)} \frac{\alpha_{l} \alpha_{v}}{\Pi_{l v}}\left(P_{l}-P_{v}\right)
$$

The product $\tau_{P}(W) \Pi_{l v}$ complies with the closure laws recalled in [21]. This sole contribution is expected to re-equilibrate phasic pressures, but this can only be checked by coupling the time evolution of the statistical fraction with the time evolution of the total phasic energies $E_{k}$ (see [20,26,30]). The internal structure of mean EOS plays a crucial role in this particular process. This remark is important since we use it in order to derive stable and physically relevant numerical methods when computing pressure relaxation source terms (see step 2 in section 3.3). Obviously, we note that we retrieve, at least in a formal way, the structure of the two-fluid six-equation models when $\tau_{P}(W)$ is set to 0 .

Eventually, as noticed in the early paper [20], we recall that we retrieve the convective structure of the Baer-Nunziato model [4] when setting: $\xi(W)=0$ (and hence $V_{\text {int }}(W)=U_{v}$ and $\left.\Pi_{\text {int }}(W)=P_{l}\right)$, or $\xi(W)=1$ (and thus $V_{\text {int }}(W)=U_{l}$ and $\Pi_{\text {int }}(W)=$ $\left.P_{v}\right)$. The reader is also refered to $[4,33,8]$ for slightly different forms of source terms within this specific class, and to $[24,7,32]$ where alternative formulations of $V_{\text {int }}(W), \Pi_{\text {int }}(W)$ are proposed.

\subsection{Main properties of the two-fluid model}

The following properties are classical (see [20]). They are recalled for sake of completeness, and they are also mandatory since we only use a sub-class associated to $(\xi(W)-1) \xi(W)=0$ in the sequel. We will also use the entropy inequality for numerical purposes.

\section{- 1- Entropy inequality}

For smooth solutions $W$ of (1) with closure laws (2), (3), (4), the governing 
equation of the entropy of the two-fluid model $\eta(W)=\sum_{k=l, v} m_{k} S_{k}$ is:

$$
\begin{array}{rlr}
\partial_{t}(\eta(W))+\partial_{x}\left(\sum_{k=l, v} m_{k} U_{k} S_{k}\right) & = & \Gamma_{l}(W)\left(\mu_{v}(W) / T_{v}-\mu_{l}(W) / T_{l}\right) \\
& + & D_{l}(W)\left(U_{v}-U_{l}\right)\left(1 /\left(2 T_{v}\right)+1 /\left(2 T_{l}\right)\right) \\
& + & \psi_{l}(W)\left(T_{v}-T_{l}\right) /\left(T_{v} T_{l}\right) \\
& + & \phi_{l}(W)\left(P_{l}-P_{v}\right)\left((1-\chi(W)) / T_{v}+\chi(W) / T_{l}\right)
\end{array}
$$

\section{- 2- Hyperbolicity}

The set of equations associated with the left-hand side of (1) has seven real eigenvalues which read:

$$
\begin{aligned}
& \lambda_{1}=V_{\text {int }}(W) \\
& \lambda_{2}=U_{v}, \quad \lambda_{3}=U_{v}-c_{v}(W), \quad \lambda_{4}=U_{v}+c_{v}(W), \\
& \lambda_{5}=U_{l}, \quad \lambda_{6}=U_{l}-c_{l}(W), \quad \lambda_{7}=U_{l}+c_{l}(W)
\end{aligned}
$$

Associated righteigenvectors span the whole space $\mathscr{R}^{7}$, if: $\left|U_{k}-V_{\text {int }}(W)\right| / c_{k} \neq 1$.

\section{- 3- Structure of the 1-field in the convective subset}

If we assume that : $\xi(W)=0$, or $(1-\xi(W))=0$, or: $\xi(W)=m_{l} /\left(m_{l}+m_{v}\right)$, then the $1-$ field associated with $\lambda_{1}=V_{\text {int }}(W)$ is linearly degenerate $(L D)$.

\section{- 4- Structure of the remaining fields in the convective subset}

Fields associated with eigenvalues $\lambda_{2,5}$ are linearly degenerate. Other fields associated with eigenvalues $\lambda_{3,4,6,7}$ are genuinely non linear $(G N L)$.

\section{- 5- Jump conditions}

If the 1-field is linearly degenerate, unique jump conditions can be written within each single field. When restricting to the case $\xi(W)=0$, these read:

$$
\left[\mathscr{I}_{1}^{p}(W)\right]_{l}^{r}=0
$$

for $p=1,5$ in the $1-2$-field, where $l, r$ states respectively refer to the left and right states apart from the $1-2$-wave, and noting $\mathscr{I}_{1}^{p}(W)$ the Riemann invariants of the $1-2$ field:

$$
\begin{array}{r}
\mathscr{I}_{1}^{1}(W)=U_{v} ; \\
\mathscr{I}_{1}^{2}(W)=m_{l}\left(U_{v}-U_{l}\right) ; \\
\mathscr{I}_{1}^{3}(W)=m_{l}\left(U_{v}-U_{l}\right)^{2}+\alpha_{v} P_{v}+\alpha_{l} P_{l} ; \\
\mathscr{I}_{1}^{4}(W)=\varepsilon_{l}+\frac{P_{l}}{\rho_{l}}+\frac{1}{2}\left(U_{v}-U_{l}\right)^{2} \\
\mathscr{I}_{1}^{5}(W)=S_{l} .
\end{array}
$$


Moreover, classical single-phase jump relations hold in the GNL fields:

$$
\begin{aligned}
{\left[\rho_{k}\left(U_{k}-\sigma\right)\right]_{l}^{r} } & =0 \\
{\left[\rho_{k} U_{k}\left(U_{k}-\sigma\right)+P_{k}\right]_{l}^{r} } & =0 \\
{\left[\left(U_{k}-\sigma\right) E_{k}+U_{k} P_{k}\right]_{l}^{r} } & =0 \\
{\left[U_{k^{\prime}}\right]_{l}^{r}=\left[\rho_{k^{\prime}}\right]_{l}^{r}=\left[P_{k^{\prime}}\right]_{l}^{r} } & =0
\end{aligned}
$$

where $\sigma$ denotes the speed of the shock wave separating left and right states, setting $k=v, k^{\prime}=l$ in the 3,4 fields (respectively $k=l, k^{\prime}=v$ in the 6,7 fields).

A dual formulation arises when $\xi(W)=1$. As recalled above, proofs are available in $[20,29]$. Other properties pertaining to the symetrizing variables and to the convexity of entropy can be found in [13], and the framework of admissible closure laws for $V_{\text {int }}(W)$ might even be broadened (see [11]). Actually, we insist that the fifth property is indeed crucial: it simply means that meaningful unsteady solutions involving (unique) shocks make sense in that case. On the contrary, if the 1-field is not linearly degenerate, one may expect that, for a given test case (for instance a Riemann problem involving at least one shock), all converged approximations will differ, depending on the inner numerical viscosity of the scheme. This is known from long, and is due to the occurence of the non-conservative products $\Pi_{\text {int }}(W) \partial_{x}\left(\alpha_{k}\right)$ and $\Pi_{\text {int }}(W) V_{\text {int }}(W) \partial_{x}\left(\alpha_{k}\right)$ in the phasic momentum and energy equations (see the work [25] for instance).

In the sequel, we will restrict to the case $\xi(W)=0$. Thus, for schemes that provide convergent approximations when the mesh is refined, we expect that approximations will converge towards the unique shock solution. It must also be recalled that in that case, exact solutions of the Riemann problem can be built, using a connection through Riemann invariants of the 1-field, which separates left and right initial states of the statistical void fraction, and the classical single-phase connection of states through the remaining LD or GNL fields. This simple technique has actually been intensively used in order to verify schemes by computing convective contributions (see the next section). 


\section{Numerical methods}

\subsection{Fractional step method}

The numerical algorithm is grounded on the fractional step strategy. This algorithm was first detailed in [28], when focusing on gas-liquid mixtures, and thus neglecting mass transfer. As a consequence, we only recall herein the main ingredients, together with a detailed formulation of the relaxation step that handles mass transfer. We recall that the present fractional step method involves several steps that comply with the whole entropy inequality (see section 2.2). For more details on steps that are not described herein, we thus refer to $[14,20,26,27,28,30]$. We nonetheless recall below very briefly all steps of the fractional step method, which contains an evolution step, which deals with convective contributions, and a relaxation step, which handles relaxation source terms (mass transfer, drag effects, energy transfer and pressure relaxation terms).

Thus the entropy-consistent fractional step method first computes approximate solutions of the homogeneous system :

- Evolution step

$$
\left\{\begin{array}{l}
\partial_{t}\left(\alpha_{v}\right)+V_{\text {int }}(W) \partial_{x}\left(\alpha_{v}\right)=0 \\
\partial_{t}\left(m_{k}\right)+\partial_{x}\left(m_{k} U_{k}\right)=0 \\
\partial_{t}\left(m_{k} U_{k}\right)+\partial_{x}\left(m_{k} U_{k}^{2}\right)+\partial_{x}\left(\alpha_{k} P_{k}\right)-\Pi_{i n t}(W) \partial_{x}\left(\alpha_{k}\right)=0 \\
\partial_{t}\left(\alpha_{k} E_{k}\right)+\partial_{x}\left(\alpha_{k} U_{k}\left(E_{k}+P_{k}\right)\right)+\Pi_{i n t}(W) \partial_{t}\left(\alpha_{k}\right)=0
\end{array}\right.
$$

starting with given initial values $W^{n}$, through the time interval $\left[t^{n}, t^{n}+\Delta t\right]$, and providing $\tilde{W}$ at the end.

Then, using initial data $\tilde{W}$, cell values $W^{n+1}$ are computed by approximating solutions of the coupled ODEs:

- Relaxation step

$$
\left\{\begin{array}{l}
\partial_{t}\left(\alpha_{v}\right)=\phi_{v}(W) \\
\partial_{t}\left(m_{k}\right)=\Gamma_{k}(W) \\
\partial_{t}\left(m_{k} U_{k}\right)=D_{k}(W)+\Gamma_{k}(W) \bar{U}_{i n t}(W) \\
\partial_{t}\left(\alpha_{k} E_{k}\right)+\Pi_{i n t}(W) \partial_{t}\left(\alpha_{k}\right)=\psi_{k}(W)+\bar{U}_{i n t} D_{k}(W)+\Gamma_{k}(W) \bar{H}_{i n t}(W)
\end{array}\right.
$$

This gives the final cell values $W^{n+1}$.

In the sequel we introduce the following mixture variables $\mathscr{M}, \mathscr{U}, \mathscr{E}$ :

$$
\begin{array}{r}
\mathscr{M}=m_{l}+m_{v} ; \\
\mathscr{U}=\left(m_{l} U_{l}+m_{v} U_{v}\right) / \mathscr{M} ; \\
\mathscr{E}=\alpha_{l} E_{l}+\alpha_{v} E_{v} .
\end{array}
$$

\subsection{Evolution step: numerical schemes and their verification}

Finite volume methods are used to compute approximations of solutions of the evolution step, which is an hyperbolic system that has no conservative form. Without any 
loss of generality, this evolution system may be rewritten in the equivalent form (even for discontinuous solutions):

$$
\left\{\begin{array}{l}
\partial_{t}\left(\alpha_{v}\right)+V_{i n t}(W) \partial_{x}\left(\alpha_{v}\right)=0 \\
\partial_{t}\left(m_{v} U_{v}\right)+\partial_{x}\left(m_{v} U_{v}^{2}\right)+\partial_{x}\left(\alpha_{v} P_{v}\right)-\Pi_{i n t}(W) \partial_{x}\left(\alpha_{v}\right)=0 \\
\partial_{t}\left(\alpha_{v} E_{v}\right)+\partial_{x}\left(\alpha_{v} U_{v}\left(E_{v}+P_{v}\right)\right)+\Pi_{i n t}(W) \partial_{t}\left(\alpha_{v}\right)=0 \\
\partial_{t}\left(m_{v}\right)+\partial_{x}\left(m_{v} U_{v}\right)=0 \\
\partial_{t}(\mathscr{M})+\partial_{x}\left(\Sigma_{k=l, v} m_{k} U_{k}\right)=0 \\
\partial_{t}(\mathscr{M} \mathscr{U})+\partial_{x}\left(\Sigma_{k=l, v}\left(m_{k} U_{k}^{2}+\alpha_{k} P_{k}\right)\right)=0 \\
\partial_{t}(\mathscr{E})+\partial_{x}\left(\Sigma_{k=l, v}\left(\alpha_{k} U_{k}\left(E_{k}+P_{k}\right)\right)\right)=0
\end{array}\right.
$$

Several Finite volume algorithms have been used up to now. The first one is a non conservative version of Rusanov scheme, which is described in detail in [20, 28]. A second algorithm was built using the VFRoe-ncv approximate Godunov solver detailed in [20], that relies on the non-conservative symetrizing variable $\alpha_{v}, U_{k}, P_{k}, S_{k}$ (see [13]). Eventually an efficient relaxation solver was proposed in $[39,12]$, that enables to handle evanescent phases. Classical second-order extensions of the latter schemes are of course possible, as underlined for instance in [14]. Obviously, many other schemes may be used, such as the relaxation solvers introduced in [2] or [3], or other approximate Riemann solvers (see [40, 43] among others). For all numerical simulations, an explicit CFL condition enforces the time step in order to stabilize numerical approximations. As emphasized in $[28,14]$, a $h^{1 / 2}$ (respectively $h^{2 / 3}$ ) rate of convergence is retrieved when the mesh size $h$ tends to 0 , when focusing on one-dimensional Riemann problems involving shocks, contact and rarefaction waves, which is precisely what is expected for first-order schemes (respectively second-order schemes) when a contact discontinuity occurs in the exact solution. One should keep in mind the fact that the two-fluid model described in section 2.1 involves two (or even three when $\xi(W)=m_{l} /\left(m_{l}+m_{v}\right)$ ) contact discontinuities. We also recall here that the accurate measure of convergence rates requires using very fine meshes (with more than $10^{5}$ cells in a 1D Riemann problem), since the global $L^{1}$ norm of the error behaves as $e_{L^{1}}(T)=C_{L D}(T) h^{1 / 2}+C_{G N L}(T) h$, so that the error associated with shock and rarefaction GNL waves may hide the rather poor convergence rate associated with LD fields. In some cases, consistency errors due to a wrong computation of -well-posed- non-conservative products may even be hidden beyond "admissible" consistent errors on too coarse meshes. The verification process confirms that Riemann problems involving "well-balanced initial data" (with respect to $\left.\lambda_{1}\right)$, that is to say couples $\left(W_{L}, W_{R}\right)$ such that:

$$
\mathscr{I}_{1}^{k}\left(W_{L}\right)=\mathscr{I}_{1}^{k}\left(W_{R}\right) \quad \text { for: } \quad k=1 \quad \text { to } 5
$$

are of particular interest (see [14]).

Eventually, we wish to emphasize once more that all these verifications are made possible due to the fact that exact unsteady solutions including complex waves with discontinuities can be exhibited, which is of course not true for some other two-fluid models, as recently pointed out in [5] for instance.

\subsection{Relaxation step: numerical schemes and their verification}

The relaxation step is split into four sub-steps. Each step treats separately velocity relaxation, pressure relaxation, temperature relaxation and mass transfer. 


\subsubsection{Velocity-pressure-temperature relaxation steps}

We recall now the three steps that enable to take velocity/pressure/temperature relaxation effects into account.

\section{Step 1: velocity relaxation}

Compute approximate solutions on each cell of:

$$
\left\{\begin{array}{l}
\partial_{t}\left(\alpha_{v} E_{v}\right)=\bar{U}_{i n t} D_{k}(W) \\
\partial_{t}\left(m_{v} U_{v}\right)=D_{k}(W) \\
\partial_{t}\left(\alpha_{v}\right)=\partial_{t}\left(m_{v}\right)=0 \\
\partial_{t}(\mathscr{M})=\partial_{t}(\mathscr{M} \mathscr{U})=\partial_{t}(\mathscr{E})=0
\end{array}\right.
$$

Step 2: pressure relaxation

Compute approximate solutions on each cell of:

$$
\left\{\begin{array}{l}
\partial_{t}\left(\alpha_{v}\right)=\phi_{v}(W) \\
\partial_{t}\left(\alpha_{v} E_{v}\right)+\Pi_{i n t}(W) \partial_{t}\left(\alpha_{v}\right)=0 \\
\partial_{t}\left(m_{v}\right)=\partial_{t}\left(m_{v} U_{v}\right)=0 \\
\partial_{t}(\mathscr{M})=\partial_{t}(\mathscr{M} \mathscr{U})=\partial_{t}(\mathscr{E})=0
\end{array}\right.
$$

Step 3: temperature relaxation

Compute approximate solutions on each cell of:

$$
\left\{\begin{array}{l}
\partial_{t}\left(\alpha_{v} E_{v}\right)=\psi_{v}(W) \\
\partial_{t}\left(\alpha_{v}\right)=\partial_{t}\left(m_{v}\right)=\partial_{t}\left(m_{v} U_{v}\right)=0 \\
\partial_{t}(\mathscr{M})=\partial_{t}(\mathscr{M} \mathscr{U})=\partial_{t}(\mathscr{E})=0
\end{array}\right.
$$

Complete details of first-order implicit Euler schemes used to approximate solutions for the first three steps together with verification test cases can be found in $[26,27,28,30]$. It must be emphasized that both partial masses $m_{k}$, and also $\mathscr{U}$ and $\mathscr{E}$ remain invariant through these three steps, which is in agreement with the continuous framework.

Moreover we note that:

- No additional constraint on the time step $\Delta t$ arises through the latter three steps in order to guarantee that statistical fractions, partial masses and internal energies remain positive when using stiffened gas EOS:

$$
\rho_{k} \varepsilon_{k}\left(P_{k}, \rho_{k}\right)=\left(P_{k}+\gamma_{k} \Pi_{k}\right) /\left(\gamma_{k}-1\right)
$$

within each phase (see $[26,28,30])$.

- The first-order implicit scheme used to compute approximations of solutions of (33) yields to the computation of a statistical void fraction per cell which is solution of a non-linear scalar equation (see [28], pages 61-62). A similar remark holds for the temperature relaxation step (34) (see [28], pages 60-61). The -implicit- update of the velocity in (32) can be achieved in a straightforward manner, using explicit formula within each cell (see [28], page 60).

- Verification test cases for the latter three steps can be found in $[26,30]$ and in [28] (pages 62-65). 


\subsubsection{Chemical potential relaxation step}

The last step takes mass transfer into account.

\section{Step 4: mass transfer}

Compute approximate solutions on each cell of:

$$
\left\{\begin{array}{l}
\partial_{t}\left(m_{v}\right)=\Gamma_{v}(W) \\
\partial_{t}\left(m_{v} U_{v}\right)=\Gamma_{v}(W) \bar{U}_{i n t}(W) \\
\partial_{t}\left(\alpha_{v} E_{v}\right)=\Gamma_{v}(W) \bar{H}_{i n t}(W) \\
\partial_{t}\left(\alpha_{v}\right)=0 \\
\partial_{t}(\mathscr{M})=\partial_{t}(\mathscr{M} \mathscr{U})=\partial_{t}(\mathscr{E})=0
\end{array}\right.
$$

We focus now on the computation of the mass transfer terms in the fourth step, assuming that:

$$
\xi(W)=0,
$$

and thus: $V_{\text {int }}(W)=U_{v}, \Pi_{\text {int }}(W)=P_{l}$, since this closure law corresponds to what will be used in the validation section.

Starting with the first three equations in (35), we get at once:

$$
\partial_{t}\left(m_{v} \varepsilon_{v}\right)=\partial_{t}\left(m_{l} \varepsilon_{l}\right)=0
$$

Hence, setting the main scalar unknown $X(t)=m_{v}(t)$, and using the fact that the mass transfer term is a function $\Gamma_{v}(W)=\Gamma\left(\rho_{l}, \rho_{v}, \varepsilon_{l}, \varepsilon_{v}\right)$, we need to solve:

$$
\partial_{t}(X(t))=\Gamma\left(\frac{\mathscr{M}^{n}-X}{\alpha_{l}^{n}}, \frac{X}{\alpha_{v}^{n}}, \frac{\left(m_{l} \varepsilon_{l}\right)^{n}}{\mathscr{M}^{n}-X}, \frac{\left(m_{v} \varepsilon_{v}\right)^{n}}{X}\right)
$$

The relaxation time scale $\tau_{\Gamma}(W)$ is frozen at time $t^{n}$ within the time step, and a simple implicit Euler scheme is used to solve the latter equation:

$$
\frac{\left(X^{n+1}-X^{n}\right)}{\Delta t}=\frac{1}{\tau_{\Gamma}\left(W^{n}\right)} \tilde{\Gamma}\left(W^{n+1}\left(X^{n+1}\right)\right)
$$

setting:

$$
\tilde{\Gamma}\left(W^{n+1}(X)\right)=\frac{X\left(\mathscr{M}^{n}-X\right)}{K_{l v}^{0} \mathscr{M}^{n}}\left(\frac{\mu_{l}\left(W^{n+1}(X)\right)}{T_{l}\left(W^{n+1}(X)\right)}-\frac{\mu_{v}\left(W^{n+1}(X)\right)}{T_{v}\left(W^{n+1}(X)\right)}\right)
$$

with:

$$
\rho_{l}^{n+1}(X)=\frac{\mathscr{M}^{n}-X}{\alpha_{l}^{n}}, \quad \rho_{v}^{n+1}(X)=\frac{X}{\alpha_{v}^{n}}
$$

and:

$$
\varepsilon_{l}^{n+1}(X)=\frac{\left(m_{l} \varepsilon_{l}\right)^{n}}{\mathscr{M}^{n}-X}, \quad \varepsilon_{v}^{n+1}(X)=\frac{\left(m_{v} \varepsilon_{v}\right)^{n}}{X}
$$

Thus we are looking for $\left.X^{n+1} \in\right] 0, \mathscr{M}^{n}$ [ solution of the equation $f\left(X^{n+1}\right)=0$, where:

$$
f(X)=X-m_{v}^{n}-\frac{\Delta t}{\tau_{\Gamma}\left(W^{n}\right)} \tilde{\Gamma}\left(\frac{\mathscr{M}^{n}-X}{\alpha_{l}^{n}}, \frac{X}{\alpha_{v}^{n}}, \frac{\left(m_{l} \varepsilon_{l}\right)^{n}}{\mathscr{M}^{n}-X}, \frac{\left(m_{v} \varepsilon_{v}\right)^{n}}{X}\right)
$$


We recall that the time step $\Delta t$ is provided by the evolution step.

\section{Proposition:}

Assume that the equation of state within each phase (for $k=l, v)$ is a stiffened gas EOS of the form:

$$
\varepsilon_{k}-q_{k}=\frac{P_{k}+\gamma_{k} \Pi_{k}}{\left(\gamma_{k}-1\right) \rho_{k}}
$$

where $q_{k}, \Pi_{k}$ are non-negative constants and $1<\gamma_{k}$. Then, whatever the ratio $\frac{\Delta t}{\tau_{\Gamma}\left(W^{n}\right)}$ is, the equation $f(X)=0$ admits a unique solution in $] 0, \mathscr{M}^{n}[$.

Proof:

When plugging stiffened gas EOS within each phase, we can check at once that:

$$
S_{k}=\left(C_{v}\right)_{k} \operatorname{Ln}\left(\left(P_{k}+\Pi_{k}\right) \rho_{k}^{-\gamma_{k}}\right)+S_{k}^{0}
$$

Hence, defining:

$$
\mu_{k}^{n}=\gamma_{k}\left(C_{v}\right)_{k}-\left(C_{v}\right)_{k} \operatorname{Ln}\left(\frac{\left(\gamma_{k}-1\right)\left(\left(m_{k} \varepsilon_{k}\right)_{n}-\Pi_{k} \alpha_{k}^{n}\right)}{\left(\alpha_{k}^{n}\right)^{1-\gamma_{k}}}\right)-S_{k}^{0}
$$

we get:

$$
\frac{\mu_{v}}{T_{v}}=\mu_{v}^{n}+\gamma_{v}\left(C_{v}\right)_{v} \operatorname{Ln}(X) \quad \text { and: } \quad \frac{\mu_{l}}{T_{l}}=\mu_{l}^{n}+\gamma_{l}\left(C_{v}\right)_{l} \operatorname{Ln}\left(\mathscr{M}^{n}-X\right)
$$

Consequently, $f(X)$ takes the following form:

$f(X)=X-m_{v}^{n}+\frac{\Delta t}{\tau_{\Gamma}\left(W^{n}\right)} \frac{X\left(\mathscr{M}^{n}-X\right)}{\mathscr{M}^{n} K_{l v}^{0}}\left(\mu_{v}^{n}-\mu_{l}^{n}+\gamma_{v}\left(C_{v}\right)_{v} \operatorname{Ln}(X)-\gamma_{l}\left(C_{v}\right)_{l} \operatorname{Ln}\left(\mathscr{M}^{n}-X\right)\right)$

For $X \in] 0, \mathscr{M}^{n}$, we may introduce a function:

$$
g(X)=\frac{f(X)}{X\left(\mathscr{M}^{n}-X\right)},
$$

and we get at once:

$$
\lim _{X \rightarrow 0^{+}} g(X)=-\infty \quad \text { and: } \quad \lim _{X \rightarrow\left(\mathscr{M}^{n}\right)^{-}} g(X)=+\infty
$$

Moreover, straightforward calculations yield, for $X \in] 0, \mathscr{M}^{n}$ :

$$
g^{\prime}(X)=\frac{X^{2}-2 X m_{v}^{n}+\mathscr{M}^{n} m_{v}^{n}}{\left(X\left(\mathscr{M}^{n}-X\right)\right)^{2}}+\frac{\Delta t}{K_{l v}^{0} \mathscr{M}^{n} \tau_{\Gamma}\left(W^{n}\right)}\left(\frac{\gamma_{v}\left(C_{v}\right)_{v}}{X}+\frac{\gamma_{l}\left(C_{v}\right)_{l}}{\mathscr{M}^{n}-X}\right)
$$

Obviously:

$$
X^{2}-2 X m_{v}^{n}+\mathscr{M}^{n} m_{v}^{n}>0
$$

since the discriminant $\delta$ is always negative:

$$
\delta=\left(m_{v}^{n}\right)^{2}-\mathscr{M}^{n} m_{v}^{n}=m_{v}^{n}\left(m_{v}^{n}-\mathscr{M}^{n}\right)=-m_{v}^{n} m_{l}^{n}<0
$$

Thus we have:

$$
\left.g^{\prime}(X)>0 \quad \text { for }: \quad \forall X \in\right] 0, \mathscr{M}^{n}[
$$


whatever $\frac{\Delta t}{\tau_{\Gamma}\left(W^{n}\right)}$ is. This in turn means that the equation: $g(X)=0$ admits at unique admissible solution $X \in] 0, \mathscr{M}^{n}$ [ since $g(X)$ is continuous and increasing in $] 0, \mathscr{M}^{n}$ [. This enables to conclude that the equation: $f(X)=0$ has the same unique solution. Even more, this proof shows that the framework of admissible couples of EOS could be broadened.

In practice, a dichotomic algorithm is used to solve the latter scalar equation $g\left(X^{n+1}\right)=$ 0 . Appendix A provides a verification test case for that particular step. It includes the description of the analytic solution, and it provides convergence rates for that step. 


\section{Validation of the two-fluid model against experimen- tal data}

Three distinct cases are considered in this section for the validation of the two-fluid model. Numerical results are compared with available experimental data. We focus here on the ability of the two-fluid model to reproduce correct pressure (acoustic, shock or rarefaction) wave propagation in air/water mixtures. Phase transition is also checked on applications characterized by high pressure variations.

\subsection{Propagation of a pressure disturbance in air/water mixtures}

During a water hammer transient, it is known that the celerity of pressure waves can be strongly modified by the presence of vapour, even in a very small quantity of the mixture (see the review [23]). In the absence of mass transfer, the speed of acoustics wave in air/water mixtures follows the following analytical relation:

$$
\frac{1}{\mathscr{M} \check{c}^{2}}=\frac{\alpha_{l}}{\rho_{l} c_{l}^{2}}+\frac{\alpha_{v}}{\rho_{v} c_{v}^{2}}
$$

still setting: $\mathscr{M}=\alpha_{l} \rho_{l}+\alpha_{v} \rho_{v}$. This well-known relation is referred as Wood speed of sound [46] or Wallis speed of sound [45]. This relation can be obtained considering two-phase flows with negligible relative motion $\left(U_{l}=U_{v}\right)$, negligible surface tension effect $\left(P_{l}=P_{v}\right)$, no mass transfer and adiabatic conditions [9]. One of the main features is that the speed of sound of the mixture can be smaller than that of either of its components. The analytical relation given in Eq. (37) is in very good agreement with the experimental data at moderate frequencies obtained by Karplus [34] (see for example [9]).

As previously shown, the two-fluid model is characterized by two pure phasic speeds of sound, i.e. $c_{l}$ and $c_{v}$, and not by a mixture speed of sound in contrast to the homogeneous models. Thus, the concept of mixture speed of sound has to be introduced in the two-fluid model framework. Then, it is relevant to check whether the influence of the presence of vapour on this mixture speed of sound can be numerically reproduced by the approximated solutions of the two-fluid model. For this purpose, an acoustic pressure disturbance is propagated in a two-phase flow mixture without temperature and mass transfer under atmospheric conditions. The numerical setup used here is detailed in the following.

The considered 1-D computational domain is $[0 ; 1]$. The initial conditions correspond to an infinitesimal pressure disturbance in a uniform two-phase flow at rest with pressure and velocity equilibria:

$$
\rho_{v}=1.2 \mathrm{~kg} \cdot \mathrm{m}^{-3}, \quad \rho_{l}=997 \mathrm{~kg} \cdot \mathrm{m}^{-3}, \quad U_{v}=U_{l}=0 \mathrm{~m} . \mathrm{s}^{-1}
$$

and:

$$
P_{v}=P_{l}=10^{5}\left(1+\delta_{P}\right) \mathrm{Pa}
$$

with the normalized pressure disturbance:

$$
\delta_{P}=\delta_{0} \exp \left(-\left(\frac{x-x_{0}}{L}\right)^{2}\right)
$$


where $\delta_{0}=10^{-3}, x_{0}=0.5 \mathrm{~m}$ and $L=1 / 20 \mathrm{~m}$. The thermodynamic constants for the stiffened gas EOS within each phase:

$$
\rho_{k}\left(\varepsilon_{k}\left(P_{k}, \rho_{k}\right)-q_{k}\right)=\left(P_{k}+\gamma_{k} \pi_{k}\right) /\left(\gamma_{k}-1\right)
$$

are:

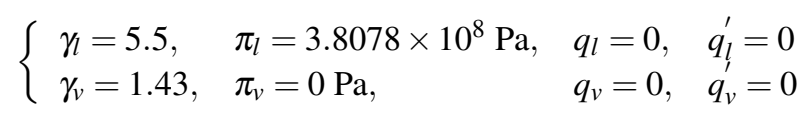

Temperature and free Gibbs energy relaxations are not taken into account as no phase transition is considered in this numerical experiment, i.e $\tau_{T}=\tau_{\Gamma}=\infty$. In order to neglect the relative motion of phases and the surface tension effect, very small pressure and velocity relaxation time scales are considered: $\tau_{P}=\tau_{U}=10^{-10} \mathrm{~s}$.

The numerical solution of this problem is symmetric with respect to $x_{0}$ : the pressure Gaussian is split into two similar structures which propagate in each direction as in the monophasic framework. The introduced speed of sound in the air/water mixture corresponds to the speed of the propagation of the initial pressure profile. This is given by $c=d / T$ where $d$ is the distance travelled by the pressure Gaussian during the time $T$. This speed of sound is measured for several initial values of $\alpha_{l}$. The corresponding numerical results are then compared to the Wallis/Wood speed of sound given in Eq. (37) and to the experimental data obtained by Karplus (see Fig. (1)). Very good agreement between the numerical "mixture" speed of sound of the two-fluid model with the Wallis/Wood speed of sound is obtained. In addition, small discrepancies are obtained with the experiments of Karplus. This demonstrates that the two-fluid model is able to reproduce pressure wave propagation in two-phase flow mixtures.

\subsection{Simpson's valve closure water hammer experiment}

The Simpson's valve closure water hammer experiment also known as Simpson's pipe column separation water hammer experiment $[42,41]$ is considered here for the validation of the two-fluid model. The experimental setup is composed by a horizontal pipe filled by a liquid water flow (see Fig. (2)). A tank is located at one boundary of the pipe.

At time zero, a valve located 36 meters away from the tank, is instantaneously closed. Due to this sudden closure, a pressure wave is generated and propagates from the valve to the tank in the horizontal pipe. Simpson [41] varied the initial velocity of the steady state liquid water fluid to affect the appearance of cavitation and the severity of the water hammer. This experiment is one of the fundamental benchmarks for two-phase flow models due to the simple geometry, initial conditions and clear water hammer initiating mechanism, i.e. a rapid valve closure. The fluid pressure is measured along the pipe at three positions: P1, P2 and P3 located at 36, 27 and 9 meters, respectively, away from the tank. The results of the simulations of the two-fluid model are compared to the experimental measurements.

One of the conclusions of Simpson [41] is that it is necessary to include the elasticity of the pipe material to obtain agreement between simulations and experiments, as the effective speed of sound is modified by the elasticity of the pipe. As in [15], the parameters of the stiffened gas EOS are chosen to recover the experimental speed of sound i.e. $c_{l} \approx 1256 \mathrm{~m} . \mathrm{s}^{-1}$ (see Table (1)). The initial conditions in the three parts of 

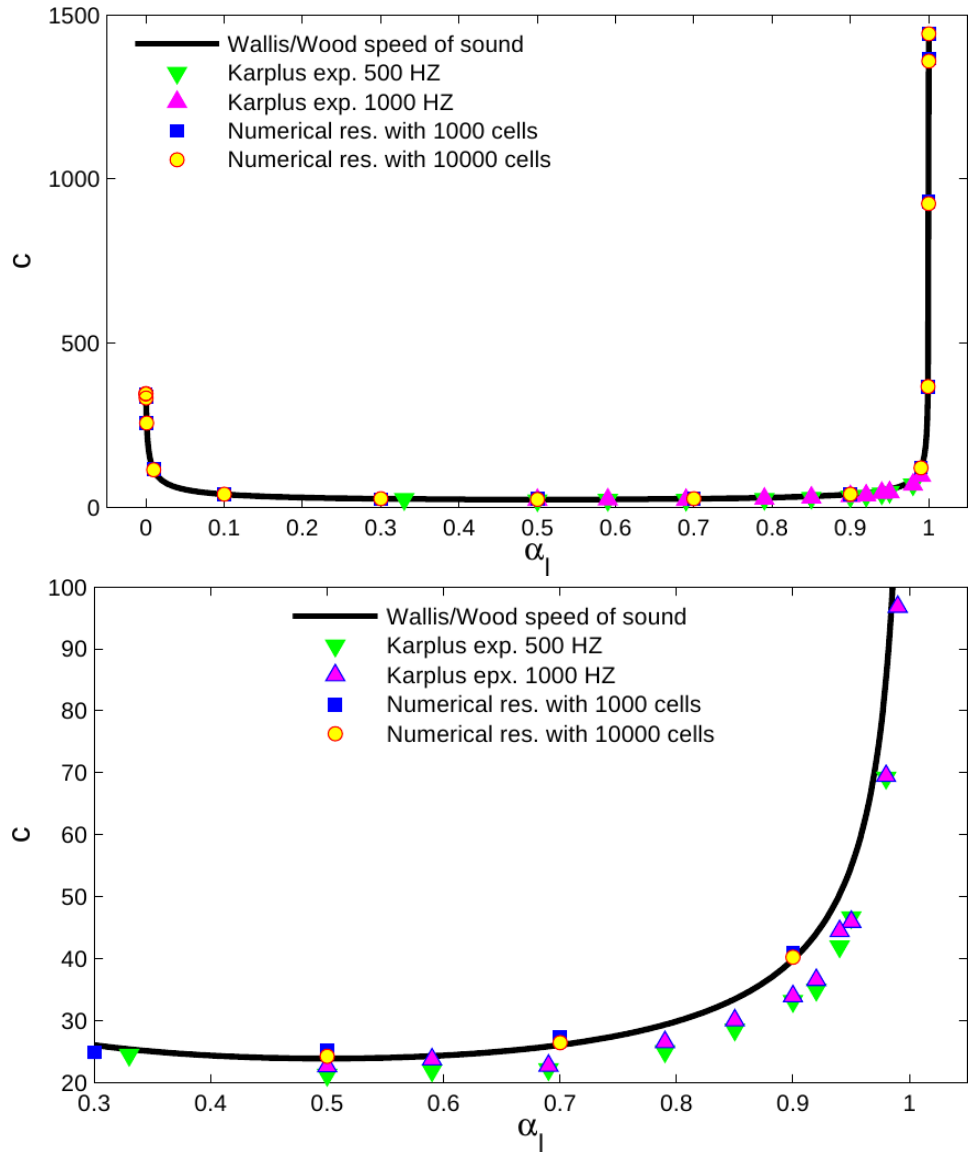

Figure 1: Speed of sound in air/water mixtures - Comparison between the Wallis/Wood speed of sound, the experimental data of Karplus [34] and the numerical approximation of the two-fluid model as a function of $\alpha_{l}$.

\begin{tabular}{|c|c|c|c|c|c|c|}
\hline Phase & $\pi(\mathrm{Pa})$ & $C_{p}(\mathrm{~J} / \mathrm{kg} / \mathrm{K})$ & $C_{v}(\mathrm{~J} / \mathrm{kg} / \mathrm{K})$ & $\gamma$ & $q(\mathrm{~J} / \mathrm{kg})$ & $q^{\prime}(\mathrm{J} / \mathrm{kg} / \mathrm{K})$ \\
\hline Liquid & 692754002.87 & 4183.00 & 1840.48 & 2.27 & -1142331.00 & 24218.87 \\
\hline Vapor & 0.00 & 1800.00 & 1344.06 & 1.34 & 2009800.00 & 1977.08 \\
\hline
\end{tabular}

Table 1: EOS parameters for liquid and vapor water for the Simpson's valve closure induced water hammer experiment. 


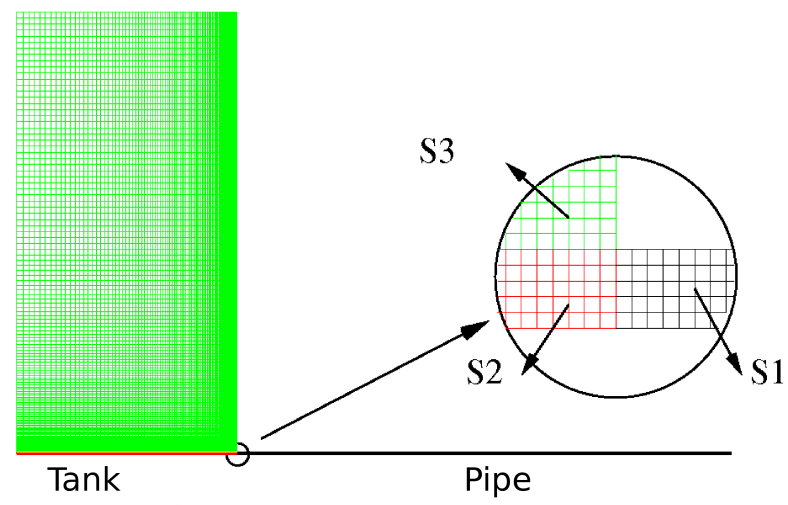

Figure 2: Sketch of the Simpson's valve closure water hammer experiment.

the computational domain represented in Fig. (2) are the following:

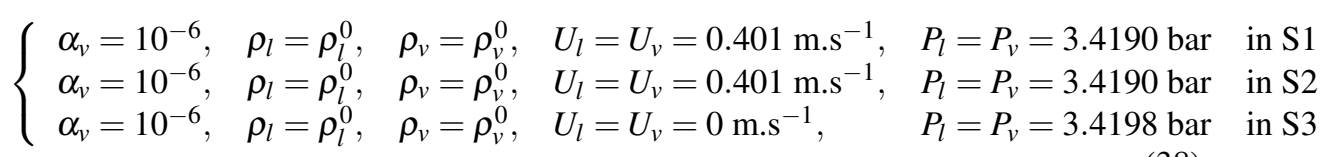

with $\rho_{l}^{0}=997.8 \mathrm{~kg} \cdot \mathrm{m}^{-3}$ and $\rho_{v}^{0}=2.52 \mathrm{~kg} \cdot \mathrm{m}^{-3}$, which corresponds to the constant temperature $T_{l}=T_{v}=296.3 \mathrm{~K}$. As the temperature is assumed to be constant during the simulation of the two-fluid model of the Simpson's water hammer, the temperature relaxation is not considered here: $\tau_{T}=\infty$. The time scales used for the other relaxations are: $\tau_{P}=\tau_{U}=10^{-7} \mathrm{~s}$ and $\tau_{\Gamma}=10^{-3} \mathrm{~s}$. All the computations presented here are obtained with the first-order Rusanov scheme in conjunction with the implicit pressure relaxation, implicit velocity and the chemical potential algorithms for the relaxation terms. First of all, four different mesh refinements are considered for grid convergence comparisons. The grid independence of the numerical results is obtained in the present test with 12000 cells in the axial direction of the pipe [36]. The present numerical solutions are obtained with the Courant number $\mathscr{C}=0.5$. The computations are performed with the Europlexus fast transient dynamics software [17].

The mean pressure $P=\alpha_{l} P_{l}+\alpha_{v} P_{v}$ history comparison between the simulation and the measurement at various locations along the pipe is given in Fig. (3). Good agreement between the computation of the two-fluid model and the experimental measurements are obtained. In particular, the different pressure peaks are well estimated with the two-fluid model.

\subsection{Canon experiment: fast decompression with sudden flashing}

The Canon experiment [37] is now considered, which is a blowdown experiment with high initial temperature $(493.15 \mathrm{~K}$ ) and pressure ( $32 \mathrm{bar}$ ). This test consists in a very fast depressurization of the water contained in a pipe $4.389 \mathrm{~m}$ long and $102.3 \mathrm{~mm}$ internal diameter by opening a full $100 \%$ break. In the Canon experiment, the pressure 
(a)

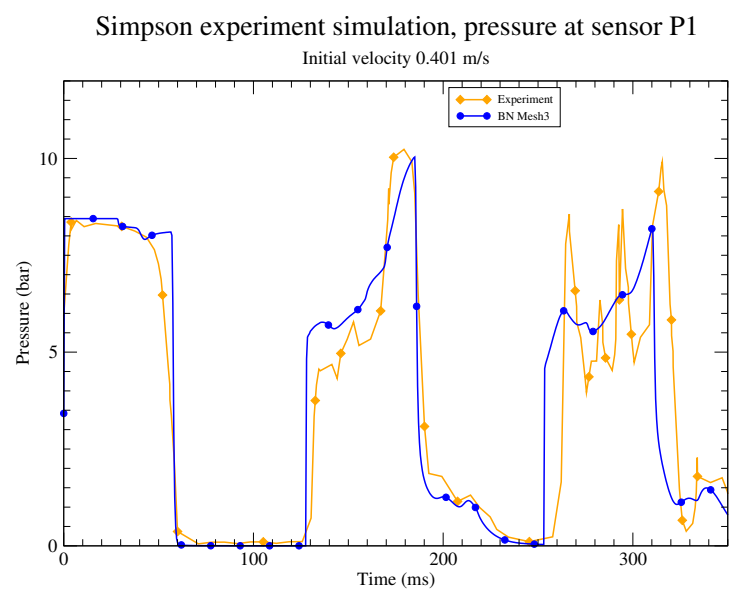

(b)

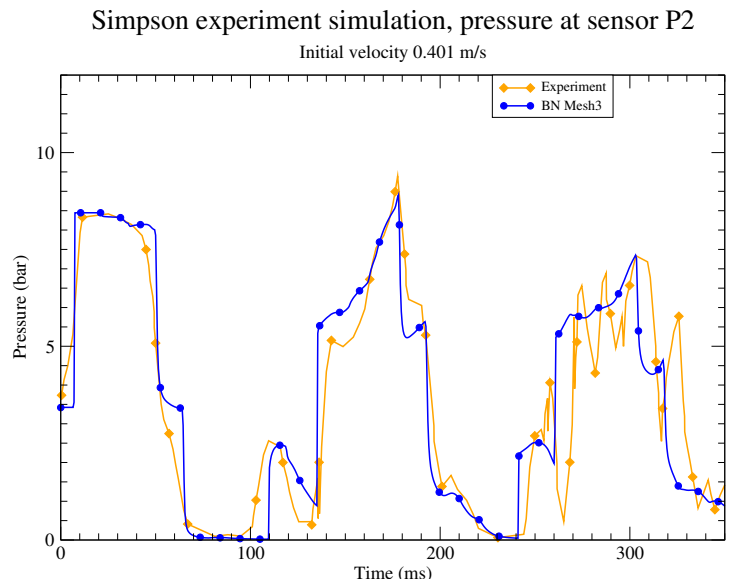

(c)

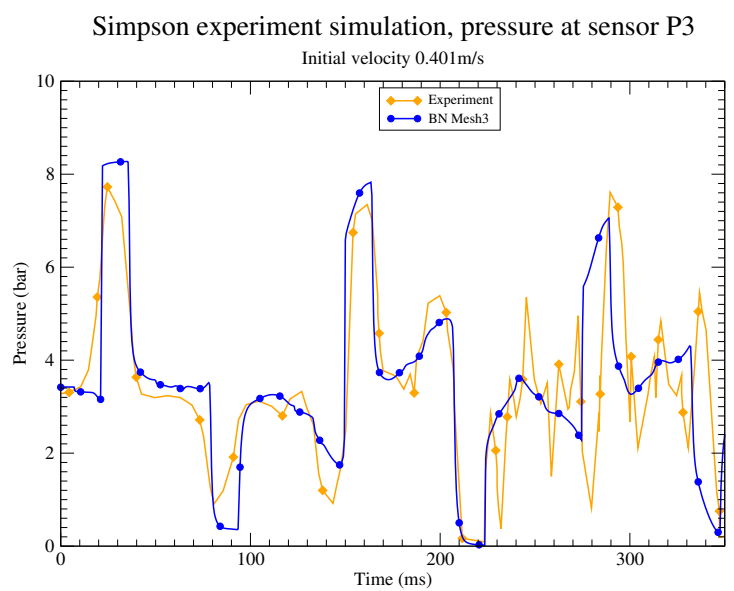

Figure 3: Mean pressure $P=\alpha_{l} P_{l}+\alpha_{v} P_{v}$ vs time at the three pressure transducers P1 (a), P2 (b) and P3 (c) in the Simpson's water hammer experiment: comparison between the two-fluid model and the experiments [48, 41]. 
is measured in several locations along the pipe whereas the void fraction is measured in only one location (cf. Figure (4)). The pressure drops from 32 bar to 1 bar in less

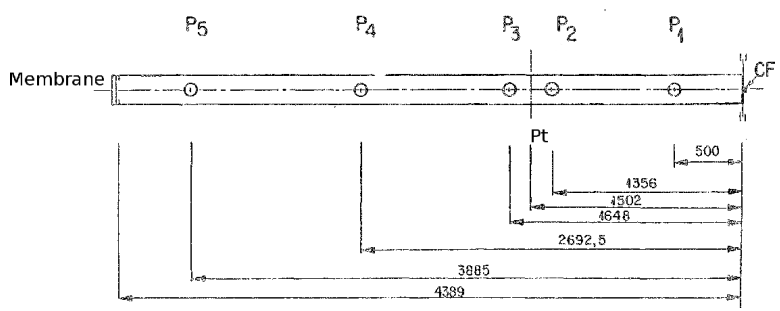

Figure 4: Schematic of the Canon test facility.

than $0.7 \mathrm{~s}$. During the first time of the transient, a rarefaction wave is generated and propagates in the pipe and the liquid water flashes.

The ability of the two-fluid model to represent a very fast depressurization with flashing is assessed via the comparison with the available experimental data [37] and the numerical computations using the HRM model based on a tabulated water EOS [35]. The computational domain consists in a horizontal pipe, closed end as boundary condition at one side and atmospheric pressure tank at the other side. As in [15], the coefficients of the stiffened gas EOS are chosen to recover the water phase diagram (cf. Table (2)). The corresponding initial conditions are defined with constant parameters

\begin{tabular}{|c|c|c|c|c|c|c|}
\hline Phase & $\pi(\mathrm{Pa})$ & $C_{p}(\mathrm{~J} / \mathrm{kg} / \mathrm{K})$ & $C_{v}(\mathrm{~J} / \mathrm{kg} / \mathrm{K})$ & $\gamma$ & $q(\mathrm{~J} / \mathrm{kg})$ & $q^{\prime}(\mathrm{J} / \mathrm{kg} / \mathrm{K})$ \\
\hline Liquid & 769317123.86 & 4670.00 & 2807.61 & 1.66 & -1359570.00 & 11671.61 \\
\hline Vapor & 0.00 & 1550.00 & 1162.00 & 1.33 & 2032350.00 & 2351.11 \\
\hline
\end{tabular}

Table 2: EOS parameters for liquid and vapor water for the Canon experiment.

in order to have the same temperature $T_{l}=T_{v}=493.15 \mathrm{~K}$ for the two phases in the entire domain:

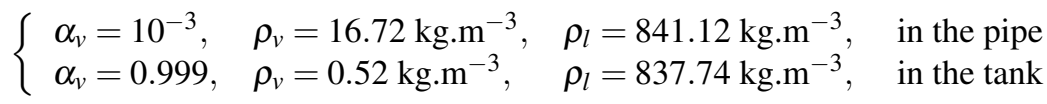

and:

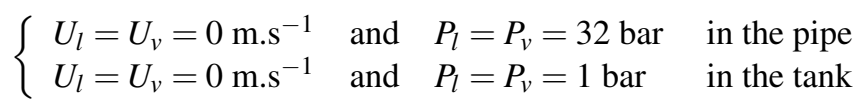

The different relaxation time scales are:

$$
\tau_{T}=0 s, \quad \tau_{U}=\tau_{P}=10^{-11} s \quad \text { and: } \quad \tau_{\Gamma}=5 \times 10^{-5} s .
$$

A preliminary grid convergence study is performed showing that results are no longer sensitive to mesh refinement beyond 4000 cells in the axial direction of the pipe [36]. The computations with the two-fluid model are performed using the first-order Rusanov scheme with the Courant number $\mathscr{C}=0.5$. The computations are still performed with the Europlexus fast transient dynamics software [17]. The experiment starts at 
time $t=0 \mathrm{~s}$ when the end of the pipe is rapidly opened.

The time evolution of the void fraction and the mean pressure $P=\alpha_{l} P_{l}+\alpha_{v} P_{v}$ at different locations is displayed in Fig. (5). The numerical results obtained with the two-fluid model are in good agreement with those obtained with the HRM model. A rarefaction wave is first generated from the break and propagates along the horizontal pipe. The pressure of the liquid water in the tube drops to the saturation pressure value. As a consequence, vaporization occurs at constant pressure: the pressure remains constant during several hundred miliseconds at sensor P5. Then, the pressure reach the atmospheric outside pressure value ( $1 \mathrm{bar}$ ). It has to be noticed that some differences are visible between numerical results and the experiments. For example, the vaporization process is numerically predicted earlier than in the experiments. 
(a)

Canon experiment simulation, void fraction of the vapour at sensor $\mathrm{Pt}$

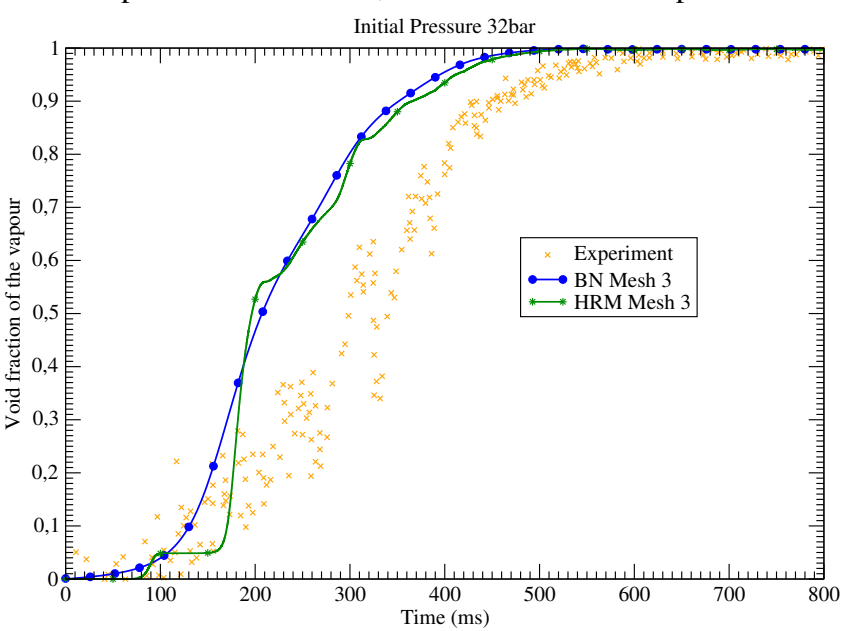

(b)

Canon experiment simulation, pressure at sensor P1

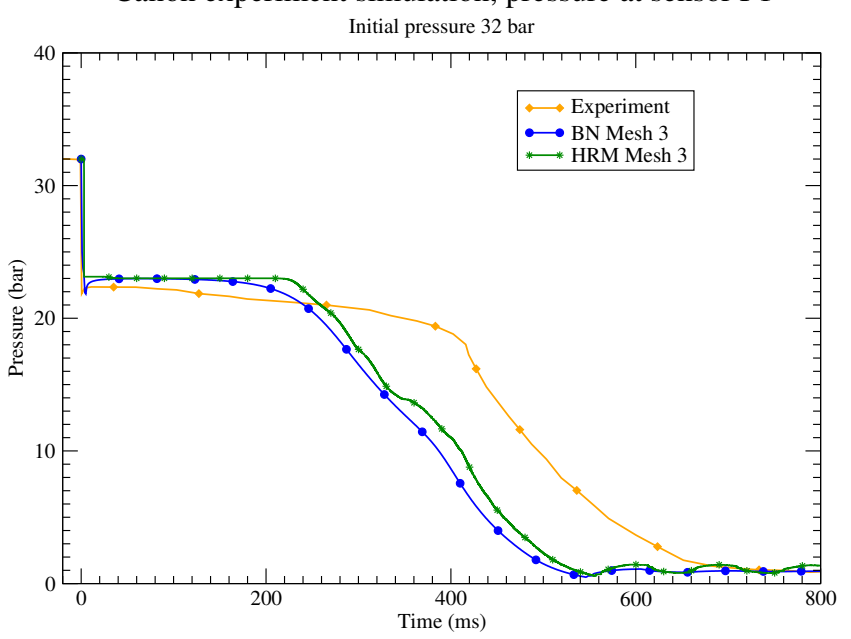

(c)

Canon experiment simulation, pressure at sensor P5 Initial pressure 32 bar

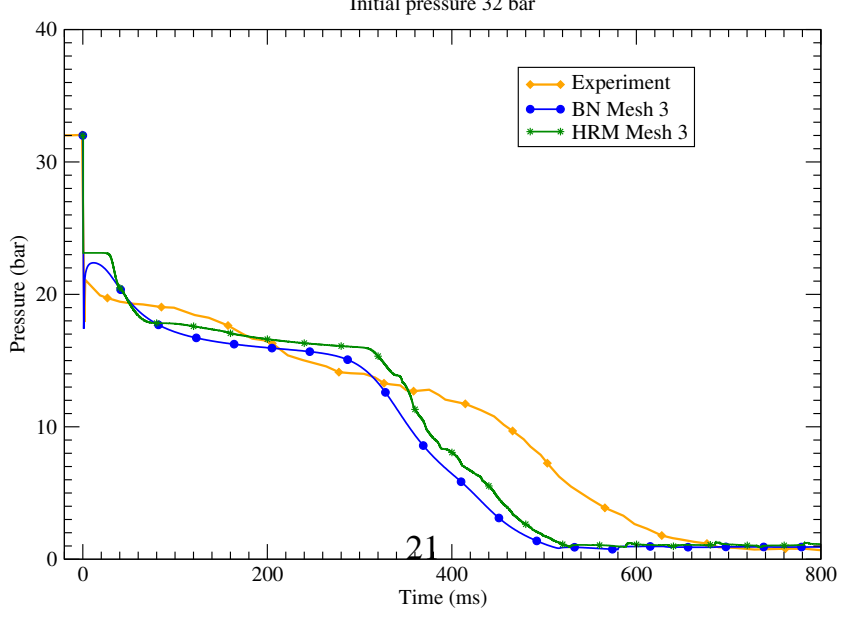

Figure 5: Vapor void fraction vs time (a) and mean pressure $P=\alpha_{l} P_{l}+\alpha_{v} P_{v}$ vs time at the first (b) and the last (c) sensor in the Canon experiment: comparison between 7-equation model, HRM and experiments [37]. 


\section{Conclusion}

A few numerical results have been reported and compared with available experimental data, while focusing on two-phase flow experiments with mass transfer involving strong rarefaction waves or shocks. The two-fluid model and its associated numerical method introduced in [28] have been recalled, and some specific details pertaining to the numerical handling of mass transfer terms and its verification have been provided. Actually, our global opinion is that:

- The two-fluid model (1) not only enables to perform meaningful unsteady computations involving sharp discontinuities, but it also seems to be a rather good candidate in order to get reasonable agreement with experimental data. This of course requires further work in order to complete the whole validation process. Beyond this, a focus on hypothetical severe accident studies would be of great interest;

- Though it is sometimes depicted as a a rather crude algorithm, the fractional step method introduced in [28] is actually a useful tool, which may of course be improved by applying for second-order or even higher-order methods for the convective part (see [43] for instance). Other alternative algorithms also seem appealant, for instance those that aim at complying with time asymptotics ([1]);

- This two-fluid model can even be used for the numerical prediction of two-phase flows with fluid-structure computations, following the basic approach described in [17] and [36]. The comparison of numerical results and experimental data in the Romander experiment confirms the feasibility, and meanwhile enhances the fact that a correct FSI approach is mandatory in order to obtain meaningful computational approximations.

\section{Acknowledgements}

During her PhD thesis, Yujie Liu received a financial support by ANRT (Agence Nationale Recherche et Technologie) through an EDF-CIFRE contract 732/2010, within the framework of SITAR project. Part of the work of Jean-Marc Hérard and Olivier Hurisse has also benefited from a financial support from the NEPTUNE project, which is a joint project between CEA, EDF, AREVA and IRSN (Institut de Radioprotection et de Sureté Nucléaire). Computational facilities were provided by EDF. Numerical simulations have been performed with the Europlexus code. 


\section{Appendix A}

- We assume that the relaxation time scale $\tau_{\Gamma}(W)$ is constant and equal to $\tau_{\Gamma}$. We seek exact solutions $X=m_{v}$ of the ODE:

$\partial_{t}(X)=\frac{-1}{\tau_{\Gamma} \mathscr{M}^{n} K_{l v}^{0}} X\left(\mathscr{M}^{n}-X\right)\left(\mu_{v}^{n}-\mu_{l}^{n}+\gamma_{v}\left(C_{v}\right)_{v} \operatorname{Ln}(X)-\gamma_{l}\left(C_{v}\right)_{l} \operatorname{Ln}\left(\mathscr{M}^{n}-X\right)\right)$

We assume that the following holds:

$$
\gamma_{v}\left(C_{v}\right)_{v}=\gamma_{l}\left(C_{v}\right)_{l}=C_{r e f}
$$

and we define a new variable $F$ and a constant $A_{r e f}$ as:

$$
F=\operatorname{Ln}\left(\frac{X}{\mathscr{M}^{n}-X}\right) \quad ; \quad A_{r e f}=\mu_{v}^{n}-\mu_{l}^{n} .
$$

Hence we look for solutions of :

$$
\partial_{t}(F)=\frac{-1}{\tau_{\Gamma} K_{l v}^{0}}\left(A_{r e f}+C_{r e f} F\right)
$$

The latter may be integrated as:

$$
F(t)=\frac{\mu_{v}^{n}-\mu_{l}^{n}}{C_{r e f}}\left(\exp \left(-\frac{C_{r e f}}{\tau_{\Gamma} K_{l v}^{0}} t\right)-1\right)+\operatorname{Ln}\left(\frac{m_{v}^{n}}{m_{l}^{n}}\right) \exp \left(-\frac{C_{r e f}}{\tau_{\Gamma} K_{l v}^{0}} t\right)
$$

which eventually provides:

$$
X(t)=\frac{\mathscr{M}^{n} \exp (F(t))}{1+\exp (F(t))}
$$

This gives the final form of the exact solution for our verification test case.

- We turn now to the verification of the relaxation step 4 using the implicit scheme (36) introduced in section 3.3.2. We consider the final time $t_{f}=10^{-3} s$, and we plot the $L^{1}$ norm $e_{L^{1}}\left(t_{f}\right)$ as a function of the time step $\Delta t$. This enables to retrieve the expected rate 1 for this test case (see figure 6). 


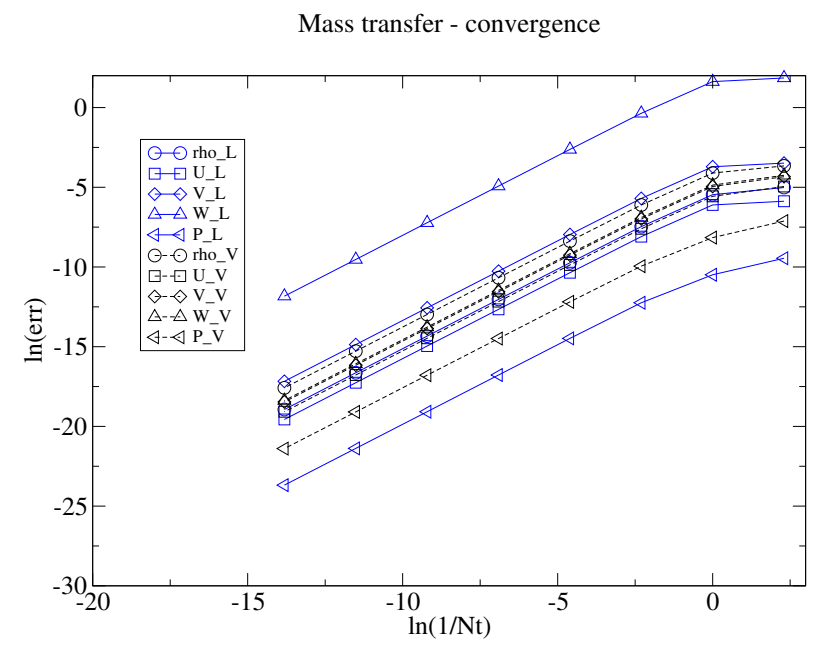

Figure 6: $L^{1}$ norm of the error $\left\|\phi-\phi^{h}\right\|$ at time $: t_{f}=10^{-3} s$ wrt $\Delta t$

\section{References}

[1] R. Abgrall, S. Dallet, An asymptotic preserving scheme for the barotropic BaerNunziato model. to appear in Springer Proceedings in Mathematics and Statistics, Vol. 78, Jürgen Fuhrmann et al (2014).

[2] A. Ambroso, C. Chalons, F. Coquel, T. Galié, Relaxation and numerical approximation of a two-fluid two-pressure diphasic model. ESAIM: Mathematical Modeling and Numerical Analysis, 43 (6), pp. 1063-1097 (2009).

[3] A. Ambroso, C. Chalons, P.A. Raviart, A Godunov-type method for the sevenequation model of compressible two-phase flow. Computers and Fluids, 54, $67-$ 91.(2012)

[4] M.R. Baer, J.W. Nunziato, A two-phase mixture theory for the deflagration to detonation transition (DDT) in reactive granular materials, International Journal of Multiphase Flow, 12(6), pp. 861-889, (1986).

[5] R. Berry, L. Zou, H. Zhao, D. Andrs, J. Peterson, H. Zhang, R. Martineau, Demonstrating seven-equation, two-phase simulation in a single pipe, two-phase reactor core and steam separator dryer, INL report INL/EXT-13-28750 (2013)

[6] C. Berthon, F. Coquel, J.M. Hérard, M. Uhlmann, An approximate solution of the Riemann problem for a realisable second-moment turbulent closure, Shock Waves, 11, pp. 245-269, (2002).

[7] W. Bo, H. Jin, D. Kim, X. Liu, H. Lee, N. Pestiau, Y. Yu, J. Glimm, J.W. Grove, Comparison and validation of multi phase closure models, Computers and Mathematics with Applications, 56, pp. 1291-1302, (2008).

[8] J.B. Bdzil, R. Menikoff, S.F. Son, A.K. Kapila, D.S. Stewart, Two phase modelling of deflagration to detonation transition in granular materials: a critical examination of modelling issues, Phys. of Fluids, 11, pp. 378-402, (1999). 
[9] C. Brennen, Fundamentals of multiphase flow, Cambridge University Press (2005)

[10] F. Coquel, T. Gallouët, P. Helluy, J.M. Hérard, O. Hurisse, N. Seguin, Modeling compressible multiphase flows, ESAIM proceedings, 40, pp. 34-50 (2013)

[11] F. Coquel, J.M. Hérard, K. Saleh, N. Seguin, A class of two-fluid two-phase flow models. In 42nd AIAA Fluid Dynamics Conference and Exhibit, AIAA paper 2012-3356, https://www.aiaa.org/, (2012).

[12] F. Coquel, J.M. Hérard, K. Saleh, N. Seguin, A robust entropy-satisfying finite volume scheme for the isentropic Baer-Nunziato model, ESAIM: M2AN, 48(1), pp.165-206 (2014).

[13] F. Coquel, J.M. Hérard, K. Saleh, N. Seguin, Two properties of two-velocity twopressure models of two-phase flows, Communications in Mathematical Sciences, 12(3), pp. 593-600 (2014).

[14] F. Crouzet, F. Daude, P. Galon, P. Helluy, J.M. Hérard, O. Hurisse, Y. Liu, Approximate solutions of the Baer Nunziato model, ESAIM proceedings, 40, pp. 63-82 (2013).

[15] F. Daude, P. Galon, Z. Gao, E. Blaud, Numerical experiments using a HLLC-type scheme with ALE formulation for compressible two-phase flows five-equation models with phase transition, Computers and Fluids, 94, 112-138, (2014).

[16] D.A. Drew, S.L. Passman, Theory of multi-component fluids, Applied Mathematical Sciences, 135, Springer, (1999).

[17] Europlexus user's manual, http://europlexus.jrc.ec.europa.eu/, Joint Research Center (JRC), Commissariat à l'énergie atomique et aux énergies alternatives (CEA) (2014).

[18] A. Forestier, S. Gavrilyuk, Criterion of hyperbolicity for nonconservative quasilinear systems admitting a partially convex conservation law. Mathematical Methods in the Applied Sciences, 34(17), 2148-2158, (2011).

[19] T. Gallouët, P. Helluy, J.M. Hérard, J. Nussbaum, Hyperbolic relaxation models for granular flows, ESAIM: Mathematical Modeling and Numerical Analysis, 44 (2), (2010).

[20] T. Gallouët, J.M. Hérard, N. Seguin, Numerical modelling of two phase flows using the two-fluid two-pressure approach, Mathematical Models and Methods in Applied Sciences, 14(5), pp. 663-700, (2004).

[21] S. Gavrilyuk, The structure of pressure relaxation terms: one-velocity case. EDF report H-I83-2014-0276-EN (2014).

[22] S. Gavrilyuk, R. Saurel, Mathematical and numerical modelling of two-phase compressible flows with micro-inertia, J. Comp. Phys., 175, pp. 326-360, (2002).

[23] M.S. Ghidaoui, M. Zhao, D.A. McInnis, D.H. Axworthy, A review of water hammer theory and practice, ASME Appl. Mech. Rev., 58, pp. 49-76, (2005). 
[24] J. Glimm, D. Saltz, D.H. Sharp, Two-phase flow modelling of a fluid mixing layer, J. Fluid Mech., 378, pp. 119-143, (1999).

[25] V. Guillemaud, Modélisation et simulation numérique des écoulements diphasiques par une approche bifluide à deux pressions. $\mathrm{PhD}$ thesis, Université Aix-Marseille, Marseille, France, 27/03/2007. http://tel.archives-ouvertes.fr/tel00169178

[26] J.M. Hérard, O. Hurisse, Schémas d'intégration du terme de relaxation des pressions phasiques pour un modèle bifluide hyperbolique, EDF report H-I81-200901514-FR, (2009).

[27] J.M. Hérard, O. Hurisse, Computing two-fluid models of compressible two-phase flows with mass transfer, AIAA paper 2012-2959, https://www.aiaa.org/, (2012).

[28] J.M. Hérard, O. Hurisse, A fractional step method to compute a class of compressible gas liquid flows. Computers and Fluids, 55, 57-69. (2012).

[29] J.M. Hérard, Y. Liu, Une approche bifluide statistique de modélisation des écoulements diphasiques à phases compressibles. EDF report H-I81-201301162-FR (2013).

[30] O. Hurisse, J.M. Hérard, Y. Liu, Note de vérification des schémas d'intégration numérique des termes sources d'un modèle diphasique à relaxation en pression. EDF report H-I83-2013-02014-FR (2014).

[31] M. Ishii, Thermofluid dynamic theory of two-phase flow, Collection de la Direction des Etudes et Recherches d'Electricité de France, Collection Eyrolles, (1975).

[32] H. Jin, J. Glimm, D.H. Sharp, Compressible two-pressure two-phase flow models, Physics letters A, 353, pp. 469-474, (2006).

[33] A.K. Kapila, S.F. Son, J.B. Bdzil, R. Menikoff, D.S. Stewart, Two phase modeling of a DDT: structure of the velocity relaxation zone, Phys. of Fluids, 9(12), pp. 3885-3897, (1997).

[34] H. Karplus, The velocity of sound in a liquid containing gas bubbles, Illinois Inst. Tech. report, number COO-248 (1958)

[35] M. Lepareux, Programme PLEXUS. Matériau "EAU". Modèle homogène équilibré, CEA report DRN/DMT 94.398, (1994).

[36] Y. Liu, Contribution à la vérification et à la validation d'un modèle diphasique bifluide instationnaire. PhD thesis, Université Aix-Marseille, Marseille, France, 11/09/2013. http://tel.archives-ouvertes.fr/tel-00864567

[37] B. Riegel, Contribution à l'étude de la décompression d'une capacité en régime diphasique. PhD thesis, Université de grenoble, (1978).

[38] J. Ristorcelli, A representation for the turbulent mass flux contribution to Reynolds stress and two-equation closures for compressible turbulence, NASA ICASE technical report 93-88, (1993). 
[39] K. Saleh, Analyse et Simulation Numérique par Relaxation d'Ecoulements Diphasiques Compressibles. Contribution au Traitement des Phases Evanescentes. PhD thesis, Université Pierre et Marie Curie, Paris, France, 26/11/2012. http://tel.archives-ouvertes.fr/tel-00761099

[40] D.W. Schwendemann, C.W. Wahle, A.K. Kapila, The Riemann problem and a high-resolution Godunov method for a model of compressible two-phase flow, $\mathrm{J}$. Comp. Phys., 212, pp. 490-526, (2006).

[41] A.R. Simpson, Large water-hammer pressures due to column separation in sloping pipes (transient cavitation). PhD thesis, University of Michigan, (1986).

[42] A. R. Simpson, E.B. Wylie, Large water-hammer pressures for column separation in pipelines 117, pp. 1310-1316, (1991).

[43] S.A. Tokareva, E.F. Toro, HLLC-type Riemann solver for the Baer-Nunziato equations of compressible two-phase flow, J. Comp. Phys., 229, pp. 3573-3604, (2010).

[44] M. Uhlmann, Etude de modèles de fermeture au second ordre et contribution à la résolution numérique des écoulements turbulents compressibles. PhD thesis, Ecole centrale de Lyon, (1997).

[45] G.B. Wallis, One Dimensional Two-Phase Flow, Mcgraw-Hill (1969)

[46] A.B. Wood, A Testbook of Sound, G. Bell and Sons Ltd., London (1930) 